\title{
The Effects of Self-induced and Therapist-assisted Lower-limb PNF Pattern Training on the Activation of Contralateral Muscles
}

\author{
Insuk Park, $\mathrm{PT}^{1)}$, Seungbum Park, PhD ${ }^{2)}$, Jaeyoung Park, $\mathrm{PhD}^{3)}$, Honghee, $\mathrm{Choi}^{\text {?), }}$ \\ Joonseo Park ${ }^{4)}$, Dongwook Han, PhD, PT $^{5)}$ \\ 1) Department of Physical Therapy, Graduate School of Silla University \\ 2) Footwear Industrial Promotion Center, Busan Economic Promotion Agency \\ 3) Department of Leisure Sports, College of Sports Science, Dong-Eui University \\ 4) Department of Physical Therapy, College of Medical and Life Science, Silla University \\ 5) Department of Physical Therapy, College of Medical and Life Science, Silla University: 100 \\ Silladaehakgil, Sasanggu, Busan 617-736, Republic of Korea. TEL: +82 51-999-6238, FAX: +82 51- \\ 999-5176,E-mail:dwhan@silla.ac.kr
}

\begin{abstract}
Purpose] The purpose of this study was to compare the effects of therapist-assisted and self-induced lower-limb PNF pattern training on the activation of contralateral muscles, with the objective of discovering whether self-led PNF pattern training can be effective. [Subjects] Six male and 15 female students participated in the experiment; after being introduced to the methods, they voluntarily signed a consent form. [Method] Members of the self-led treadmill exercise group went through three sets of extension, abduction, and internal rotation-the lower-limb pattern of PNF training-according to a researcher's spoken instructions. The other group went through three sets of the same exercises, receiving direct resistance training from a therapist. A surface EMG was used for measurement, and the average values of three measurements were used for both groups. [Result] In the treadmill group, muscular activation of the opposite-side lower limbs showed a significant difference. There were also changes in muscular activation in the PNF pattern group conducted by a therapist. It was found that muscular activation of the gastrocnemius was higher in the group that received training from a therapist, while semitendinosus activation was higher in the self-led treadmill group. [Conclusion] In conclusion, these findings suggest that PNF training on a treadmill can be effective in promoting muscular activation of the contralateral semitendinosus, while therapist-led PNF training promotes muscular activation of the gastrocnemius. Overall, lower-limb PNF pattern training of one side of the body can be an effective treatment method for promoting muscular activation of the opposite side. Key words: PNF pattern, Treadmill, Muscular activation
\end{abstract}

(This article was submitted May 1, 2012, and was accepted Jun. 20, 2012)

\section{INTRODUCTION}

Proprioceptive neuromuscular facilitation (PNF) is a treatment method that uses unique spiral or diagonal patterns to promote neuromuscular function. PNF stimulates proprioception locations, such as muscle spindles, which respond to changes in muscular length or tension. It is known to be effective in inducing maximum motor unit response and increasing the coordination of muscles through neuromuscular stimulation $^{1)}$. Typically, PNF aims to enhance bodily functions by stimulating the proprioception of muscles and tendons. To fortify the muscular strength of weakened parts or to work on a weaker side of the body through resistance and patterns, PNF is also used to induce muscle contraction on the weak side and to promote muscular strength through resistance against the strong side ${ }^{2}$. Treatment methods that aim to reinforce the weaker side of the body by applying exercise to the stronger side are referred to as irradiation ${ }^{1)}$. To create irradiation effects through resistance, muscular exercise is performed for one sound body part so as to promote muscular activation of other parts. In the case of PNF, resistance or stronger patterns of movement are used on healthy body parts in order to enhance the strength of contralateral paralyzed or weakened muscles that are hard to approach directly ${ }^{3)}$. The irradiation effect is based on research findings that an exercise on one side of the body affects the opposite side, and is also known as the cross-training effect or contralateral effect. Its mechanism is not clearly known, but the basic concept is that steady exercise of limbs on one side can have an influence on the other side ${ }^{4}$. Thus, for hemiplegia patients who cannot voluntarily move their limbs on one side, the contralateral effect is widely pursued by exercising the strong side to promote muscular power and motor function on the weak side. This approach is reported to have positive effects ${ }^{5}$. Shima et al. (2002) demonstrated that resistance training with electromyograms on one side of the body increased muscular strength of the other side; similarly6), Bemben and Murphy (2001) reported that short- 
term resistance training on one side enhanced the strength of the muscles that were not trained ${ }^{7)}$. Ress et al. (2007) argued that PNF training increased the maximum voluntary contraction (MVC) of the ankle by $26 \%$. Although some studies have shown that PNF can be effective in promoting muscular strength, its drawback is that the training requires a therapist for effective movements of resistance and patterns ${ }^{8)}$ Meanwhile, adequate intensity and repetition is essential for effective intervention therapy ${ }^{9}$, and the training routine should consist of movements during daily life so that the patient exercises for many hours a day. Considering this, the most efficient approach is to help patients understand their own problems and progressively participate in their own treatment. In terms of methods, it is most effective to educate patients so that they can exercise by themselves at home ${ }^{10)}$. Therefore, in conducting cross-training through PNF on the body parts that are incapable of voluntary movement, it is important that a patient understand his own problems and learn self-training methods to actively participate in the treatment. However, only a few studies have been undertaken to compare the effects of therapist-led PNF pattern training with those of self-motivated PNF pattern training. In this context, this study examines the treatment results of two cases. A therapist provided PNF pattern and resistance training in one case, while in the other, patients exercised by themselves using the same pattern training. The objective was to examine whether self-led PNF pattern training can be effective.

\section{SUBJECTS AND METHODS}

The research participants were selected from students of S university in Busan, Republic of Korea, who had control over independent weight-bearing of the lower limbs on both sides and no abnormalities in the muscular or nervous systems. Six male and 15 female students participated in the experiment. They were introduced to the experiment after being informed of the procedures of the experiment and voluntarily signing a consent form. Three male and seven female students were randomly selected for a treadmill exercise group; the others formed a group that would receive training from a therapist. The mean age of the participants was 21 , their mean height was $163.52 \mathrm{~cm}$, and their mean weight was $58.57 \mathrm{~kg}$. Members of the treadmill exercise group went through three sets of extension, abduction, and internal rotation-the lower limb pattern of PNF training-according to a researcher's spoken instructions. Members of the other group went through three sets of the same exercises, receiving direct resistance training from a therapist. Members of the treadmill exercise group pushed a running belt with one foot to perform PNF pattern training of the lower limb, with the movement of the belt working as resistance. To ensure safety, participants were asked to hold the treadmill bar, and tables were put next to the members of the therapist-induced exercise group to ensure a stable posture. For analysis, the average values of three measurements were used for both groups. A surface electromyogram (TeleMyo DTS, Noraxon U.S.A. Inc., Scottsdale, AR, USA) was used for measurement, and as Jung (2009) suggested $^{11)}$, the electrodes were attached to the muscle belly of the tibialis anterior, the vastus lateralis, the gastrocnemius, and the semitendinosus. To reduce measurement error, the electrode's location was shaved and cleansed with alcohol. Electrodes were firmly attached to the skin to prevent noise coming from their movement during the PNF pattern training. For EMG analysis, EMG normalization was conducted for each muscle in units of \%MVIC (maximal voluntary isometric contraction), as shown below: NorEMG $(\% \mathrm{MVIC})=\mathrm{EMG}_{\mathrm{m} /} \mathrm{EMG}_{\max } \times 100$. Here, NorEMG represents the normalized $\mathrm{EMG}$ value of each muscle, $\mathrm{EMG}_{\mathrm{m}}$ indicates the EMG value of each muscle measured during the training, and $\mathrm{EMG}_{\max }$ stands for the EMG value of the maximum MVIC of each muscle. A Shapiro-Wilk normality test was conducted to verify the normality of the 21 subjects, and as the normality could not be verified, a nonparametric test was also done. To compare muscular activation in the two groups, a Mann-Whitney U test was conducted; a KruskalWallis $\mathrm{H}$ test was performed to identify types of muscles that became active during lower-limb PNF pattern training, and a Mann-Whitney $U$ test was performed as a post hoc test. SPSS for Windows (ver. 19.0) was used for statistical analysis, and the significance level was set at $\alpha=0.05$.

\section{RESULTS}

For the participants who performed PNF pattern training on a treadmill, muscular activation of the opposite-side lower limbs showed a significant difference. The post-hoc test results showed that muscular activation of the vastus lateralis was higher than that of the tibialis anterior $(p<0.01)$, gastrocnemius $(p<0.01)$, and semitendinosus $(p<0.01)$. Muscular activation also showed changes in the group that received PNF pattern training from a therapist, and the post hoc test showed that muscular activation of the vastus lateralis was higher than that of the tibialis anterior $(p<0.05)$ and semitendinosus $(p<0.01)$ and that that of the gastrocnemius was higher than that of the tibialis anterior $(p<0.01)$ and semitendinosus $(p<0.01)$ (Table 1). In both groups, the impact of PNF training on contralateral muscular activation was examined; muscular activation of the anterior tibialis and the vastus lateralis was higher for the group that performed the treadmill exercise, but the difference was statistically insignificant. In comparison, muscular activation of the gastrocnemius was higher in the group that received training from a therapist $(\mathrm{p}<0.05)$, and that of the semitendinosus was higher in the group that performed the treadmill exercise $(\mathrm{p}<0.05)$ (Table 2$)$.

\section{DISCUSSION}

In cross education, or cross-training, muscular exercise on one side of the body affects muscular activation of the other side. Many related studies have been undertaken recently, as cross-training can strengthen the muscular power of body parts that are hard to train directly ${ }^{12}$. These studies have shown that muscular and endurance training for limbs of one side can enhance the muscular power, function, and endurance of the limbs on the other side due to the interlateral 
Table 1. The muscle activation of the low extremity on the opposite side in exercise on a treadmill or led by a therapist

\begin{tabular}{llcc}
\hline Variables & & Mean \pm SD & Mean Rank \\
\hline \multirow{3}{*}{ Treadmill** } & Anterior Tibialis & $18.9 \pm 11.8$ & 16.8 \\
& Vastus Lateralis $\dagger$ & $63.4 \pm 42.0$ & 32.6 \\
\cline { 2 - 4 } & Gastrocnemius & $16.7 \pm 14.2$ & 14.4 \\
& Semitendinosus & $23.9 \pm 22.0$ & 18.3 \\
\hline \multirow{2}{*}{ Therapist**** } & Anterior Tibialis & $14.9 \pm 10.8$ & 16.1 \\
& Vastus Lateralis $\dagger$ & $45.5 \pm 18.7$ & 33.7 \\
\cline { 2 - 4 } & Gastrocnemius\# & $43.4 \pm 37.1$ & 29.3 \\
& Semitendinosus & $8.8 \pm 4.7$ & 10.9 \\
\hline
\end{tabular}

Unit: $\% * p<0.01,{ }^{* *} p<0.001 .{ }^{\dagger}$ : The muscle activation of the vastus lateralis was more significant than that of the anterior tibialis $(\mathrm{p}<0.01)$, gastrocnemius $(\mathrm{p}<0.01)$, and semitendinosus $(\mathrm{p}<0.01)$. The muscle activation of the vastus lateralis was more significant than that of the anterior tibialis $(\mathrm{p}<0.05)$ and semitendinosus $(\mathrm{p}<0.01)$. \#: The muscle activation of the gastrocnemius was more significant than that of the anterior tibialis $(\mathrm{p}<0.01)$ and semitendinosus $(\mathrm{p}<0.01)$.

Table 2. The effects on the muscle activation of the low extremity on the opposite side, according to exercise type

\begin{tabular}{llccc}
\hline Variables & & Mean \pm SD & Mean Rank & Rank Sum \\
\hline \multirow{2}{*}{ Anterior Tibialis } & Treadmill & $18.9 \pm 11.8$ & 12.1 & 121.0 \\
& Therapist & $14.9 \pm 10.8$ & 10.0 & 110.0 \\
\hline \multirow{2}{*}{ Vastus Lateralis } & Treadmill & $63.4 \pm 42.0$ & 12.1 & 121.0 \\
& Therapist & $45.5 \pm 18.7$ & 10.0 & 110.0 \\
\hline \multirow{2}{*}{ Gastrocnemius* } & Treadmill & $16.7 \pm 14.2$ & 7.9 & 79.0 \\
& Therapist & $43.4 \pm 37.1$ & 13.8 & 152.0 \\
\hline \multirow{2}{*}{ Semitendinosus*** } & Treadmill & $23.9 \pm 22.0$ & 15.0 & 150.0 \\
& Therapist & $8.8 \pm 4.7$ & 7.4 & 81.0 \\
\hline
\end{tabular}

Unit: $\% .{ }^{*} \mathrm{p}<0.05,{ }^{* *} \mathrm{p}<0.01$.

transfer of training ${ }^{13)}$ and can enhance their performance function ${ }^{14)}$. Muun et al. (2004) conducted a meta-analysis to examine the effect of training on one side on muscular strength of the other side and reported that cross-training can be effective. Later, Muun et al. (2005) conducted additional research to verify that there is a cross-training effect on the same types of muscles ${ }^{15}$. Zhou (2000) analyzed previous studies to examine the effect of cross-training and argued that the muscular power of the limbs on the other side can be strengthened, depending on the intensity of the exercise ${ }^{16)}$. Lagerquist et al. (2006) suggested that the effect of crosstraining would come from the brain region of the central nervous system, not the spinal cord ${ }^{17)}$. Munn et al. (2005) explained that muscular training on one side of the body can enhance the muscular power of the limbs on the other side because it involves more motor units and the activation increases in the central nervous system ${ }^{15}$. Similarly, Hortobagyi et al. (2003) and Kristeva et al. (1991) reported that the cross-training effect is controlled at the cortical level; they explained that the cross-training effect occurs as the sensory motor faculty of both sides becomes active ${ }^{18,19)}$. The cross-training effect is applied in PNF training to promote muscular power of the weak side and has proven effective. Recently, a study was conducted among people with suffi- cient ability to voluntarily control their muscles; a PNF leg pattern was applied to the lower limbs of one side, and the muscular power of the leg on the other side was measured, with the result showing a significant increase in muscular power and endurance ${ }^{20)}$. In another study, people performed upper-limb PNF patterns-flexion, abduction, and external rotation-and a lifting pattern for both upper limbs; the results showed that the PNF patterns significantly increased muscular activation of the lower limbs on the other side ${ }^{21)}$. In our study, it was shown that lower-limb PNF pattern training of one side on a treadmill increased muscular activation of the vastus lateralis on the other side, and in the group that received training from a therapist, muscular activation of the vastus lateralis and the gastrocnemius increased. That is to say, PNF pattern training on the lower limbs of the strong side increased muscular activation of the same muscles on the other side. Most of the previous studies examined the impact of PNF training led by a therapist. However, recent studies have shown that self-driven exercise can have a positive impact on enhancing bodily functions and that there is growing interest in applying self-exercise to PNF training. In this context, the present study examined whether there is a difference in exercise effects between a group that performed self-exercise on a treadmill and a 
group that received training from a therapist. Among the examined muscles, the gastrocnemius showed greater muscular activation in the group that received training from a therapist, while the semitendinosus showed higher muscular activation in the group that performed the treadmill exercise. This can be attributed to the parts where resistance was applied. In leading the exercise, a therapist applies resistance to the sole, strongly stimulating the gastrocnemius, which is involved in knee and ankle joint plantar flexion. Accordingly, the cerebral cortex transmits stimulation to activate the same muscle on the opposite side. In comparison, the participants in the treadmill exercise group kept their soles on the running belt and pushed on it, keeping the knee joint slightly bent; in this posture, the semitendinosus is strongly stimulated, increasing muscular activation of the same muscles on the opposite side. The experiment results suggest that PNF pattern training can increase tension of the same muscles on the other side but that different types of muscles are stimulated, depending on whether the patient exercises on a treadmill or receives training from a therapist. For this reason, it will be necessary to choose an adequate exercise method, depending on the patient's condition and needs. In conclusion, these findings suggest that PNF training on a treadmill can be effective in promoting muscular activation of the semitendinosus, while therapist-led PNF training can promote muscular activation of the gastrocnemius. Overall, it can be said that lower-limb PNF pattern training of one side of the body can be an effective treatment method for promoting muscular activation of the opposite side.

\section{REFERENCES}

1) Knott M, Voss DE: Proprioceptive Neuromuscular Facilitation: Patterns and Techniques; New York: Paul B Haeber, 1956

2) Lee MK, Kim JM, Park HK: The effects of proprioceptive neuromuscular facilitation leg patterns on the muscle activation of neck flexors. J Korean Acad Univ Trained Phys Therapists, 2008, 15: 46-53.

3) Adler S, Beckers D, Buck M: PNF in practice (3rd ed), Berlin: Springer, 2008 .

4) Munn J, Herbert RD, Gandevia SC: Contralateral effects of unilateral resistance training: a meta-analysis. J Appl Physiol, 2004, 96: 1861-1866. [Medline] [CrossRef]

5) Fujiwara T, Hara Y, Chino N: The influence of non-paretic leg movement on muscle action in paretic leg of hemiplegic patients. Scand J Rehabil Med, 1999, 31: 174-177. [Medline] [CrossRef]

6) Shima N, Ishida K, Katayama $K$, et al. Cross education of muscula strength during unilateral resistance training and detraining. Eur J Appl Physiol, 2002, 86: 287-294. [Medline] [CrossRef]

7) Bemben MG, Murphy RE: Age related neural adaptation following short term resistance training in women. J Sports Med Phys Fitness, 2001, 41: 291-299. [Medline]

8) Rees SS, Murphy AJ, Watsford ML, et al.: Effects of proprioceptive neuromuscular facilitation stretching on stiffness and force-producing characteristics of the ankle in active women. J Strength Cond Res, 2007, 21: 572-577. [Medline]

9) Coote S, Stokes EK: Effect of robot-mediated therapy on upper extremity dysfunction post-stroke-a single case study. Physiotherapy, 2005, 91: 250-256. [CrossRef]

10) Song JY: Effects of a group self exercise program on quality of life and motor functions after stroke. J Korean Acad Univ Trained Phys Therapists, 2008, 15: 20-29.

11) Jung HS: A study of muscle activities and gait analysis between young adults and elderly people according to gait speed in level walking. Daegu University, Dissertation of Doctorate Degree, 2009

12) Carroll TJ, Herbert RD, Munn J, et al. Contralateral effects of unilatera strength training: evidence and possible mechanisms. J Appl Physiol, 2006, 101: 1514-1522. [Medline] [CrossRef]

13) Farthing JP, Borowsky R, Chilibeck PD, et al.: Neurophysiological adaptations associated with cross-education of strength. Brain Topogr, 2007, 20: 77-88. [Medline] [CrossRef]

14) Hortobágyi $T$, Scott $K$, Lambert et al.: Cross-education of muscle strength is greater with stimulated than voluntary contractions. Motor Control, 1999, 3: 205-219. [Medline]

15) Munn J, Herbert RD, Hancock MJ, et al.: Training with unilateral resistance exercise increases contralateral strength. J Appl Physiol, 2005, 99: 1880-1884. [Medline] [CrossRef]

16) Zhou S: Chronic neural adaptations to unilateral exercise: Mechanisms of cross-education. Exerc Sport Sci Rev, 2000, 28: 177-184. [Medline]

17) Lagerquist $\mathrm{O}$, Zehr EP, Docherty D: Increased spinal reflex excitability is not associated with neural plasticity underlying the cross education effect. J Appl Physiol, 2006, 100: 83-90. [Medline] [CrossRef]

18) Hortobágyi T, Taylor JL, Petersen NT, et al.: Changes in segmental and motor cortical output with contralateral muscle contractions and altered sensory inputs in humans. J Neurophysiol, 2003, 90: 2451-2459. [Medline] [CrossRef]

19) Kristeva R, Cheyne D, Deecke L: Neuromagnetic fields accompanying unilateral and bilateral voluntary movements: topography and analysis of cortical sources. Electroencephalogr Clin Neurophysiol, 1991, 81: 284-298. [Medline] [CrossRef]

20) Kofotolis N, Kellis E: Cross-training effects of a proprioceptive neuromuscular facilitation exercise programme on knee musculature. Phys Ther Sport, 2007, 8: 109-116. [CrossRef]

21) Kim KH: Effect of proprioceptive neuromuscular facilitation applied to the unilateral upper extremity on the muscle activation of contralateral lower extremity. Eulji University, Master thesis, 2005. 